\title{
EpCAM and solid tumour fractionation
}

\section{Jane E. Visvader \& Geoffrey J. Lindeman}

We would like to thank Olivier Gires, Christoph Klein and Patrick A. Baeuerle for their correspondence on our Review (Cancer stem cells in solid tumours: accumulating evidence and unresolved questions. Nature Rev. Cancer 8, 755-768 $(2008))^{1}$, which raises some important issues (On the abundance of EpCAM on cancer stem cells. Nature Rev. Cancer 15 Jan 2009 (doi:10.1038/nrc2499-c1) $)^{2}$.

We agree that epithelial cell adhesion molecule (EpCAM) has potential as a cancer stem cell (CSC) marker and that its name is best standardized to EpCAM (other names include ESA and Ber-EP4). As an almost pan-epithelial marker in some tissues, such as colon, it is difficult to estimate the importance of EpCAM as a marker of CSCs in solid tumours versus its role as a more general epithelial surface marker. Nevertheless, it is likely to be useful in the purification of some CSCs originating in epithelial tumours. Further studies are needed to define the status and role of EpCAM as a CSC marker, as the existing data are preliminary for breast and colorectal CSCs but appear more compelling for the pancreatic CSC. For pancreatic tumours, CD44, CD24 and EpCAM (referred to as ESA) together conferred a $>100$-fold increase in tumorigenic potential ${ }^{3}$. In the case of breast tumours, EpCAM led to considerable enrichment of CSCs in one breast tumour ${ }^{4}$, but was less useful in a second tumour, as both the EpCAM-positive and EpCAM-negative fractions from this tumour had tumorigenic activity. It is presumed that EpCAM did not lead to further enrichment in the other seven tumours examined. Interestingly, EpCAM is commonly used for the purification of circulating breast tumour cells ${ }^{5,6}$. In the context of colorectal CSCs, EpCAM was expressed on almost all tumour cells (both $\mathrm{CD}_{133^{+}}$and CD133-). In these studies, EpCAM expression was used to confirm that the high proportion of CD133cells in xenografts corresponded to cancer cells of human epithelial origin ${ }^{7,8}$.

Jane E. Visvader \& Geoffrey J. Lindeman are at the VBCRC Laboratory, The Walter and Eliza Hall Institute of Medical Research, Melbourne, Victoria 3050, Australia and the Department of Medical Oncology, Royal Melbourne Hospital, Parkville, Victoria 3050, Australia. Correspondence to J.E.V. e-mail:visvader@wehi.edu.au

Published online 9 January 2009

1. Visvader, J. E. \& Lindeman, G. J. Cancer stem cells in solid tumours: accumulating evidence and unresolved questions. Nature Rev. Cancer 8, 755-768 (2008).

2. Gires, O. Klein C. \& Baeuerle, P. A. On the abundance of EpCAM on cancer stem cells. Nature Rev. Cancer 15 Jan 2009 (doi: 10.1038/nrc2499-c1).

3. Li, C. et al. Identification of pancreatic cancer stem cells. Cancer Res. 67, 1030-1037 (2007).

4. Al-Hajj, M. Cancer stem cells and oncology therapeutics. Curr. Opin. Oncol. 19, 61-64 (2007).

5. Cristofanilli, M. et al. Circulating tumor cells, disease progression, and survival in metastatic breast cancer N. Engl. J. Med. 351, 781-791 (2004).

6. Nagrath, S. et al. Isolation of rare circulating tumour cells in cancer patients by microchip technology. Nature 450, 1235-1239 (2007).

7. O'Brien, C. A., Pollett, A., Gallinger, S. \& Dick, J. E. A human colon cancer cell capable of initiating tumour growth in immunodeficient mice. Nature $\mathbf{4 4 5}$ 106-110 (2007).

8. Ricci-Vitiani, L. et al. Identification and expansion of human colon-cancer-initiating cells. Nature $\mathbf{4 4 5}$, 111-115 (2007). 